\title{
The darmstadtium cornerstone
}

\section{Dieter Ackermann explains why element 110 occupies a significant place in the superheavy corner of} the periodic table.

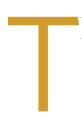
he first isotope of darmstadtium to be synthesized was ${ }^{269} \mathrm{Ds}$, in November 1994, through a cold fusion reaction between ${ }^{62} \mathrm{Ni}$ and ${ }^{208} \mathrm{~Pb}$ (ref. 1). The concept of cold fusion, where a nucleus is formed in a fusion process at low excitation energy, had already been exploited successfully in the hunt for superheavy nuclei by the team working at the accelerator laboratory GSI in Darmstadt, Germany - the city that gave its name to this element.

The team at GSI had already successfully synthesized elements 107 (bohrium), 108 (hassium) and 109 (meitnerium) in 1981, 1984 and 1982, respectively. Ten years had elapsed, during which the group had improved the efficiency of their experimental set-up to cope with ever-lower probabilities for the synthesis of ever-heavier elements. To attempt the synthesis of element 110 , it was crucial to first measure the 'excitation function' - a production probability as a function of energy - of the hassium isotope ${ }^{266} \mathrm{Hs}(Z=108)$. An extrapolation then allowed the team to successfully predict the correct energy needed for the synthesis of darmstadtium.

The targeted isotope ${ }^{269} \mathrm{Ds}$ was indeed produced by fusion between ${ }^{62} \mathrm{Ni}$ and ${ }^{208} \mathrm{~Pb}$, followed by 'cooling down' of the fused system through a one-neutron emission. But there was more: the team had enough time to change the projectile to ${ }^{64} \mathrm{Ni}$ and synthesize a second isotope, ${ }^{271} \mathrm{Ds}$, and to then go one step further. They exchanged the ${ }^{208} \mathrm{~Pb}$ foil that had served as the target for the previous two reactions to a ${ }^{209} \mathrm{Bi}$ one, featuring one more proton, thus producing element 111 (roentgenium) in the same exciting run.

An earlier attempt to synthesize ${ }^{271} \mathrm{Ds}$ at higher beam energy had failed in 1985, despite having applied a beam dose that was three times higher ${ }^{2}$. One of the reasons for the choice of the higher energy had been a concept called 'extra push', according to which an additional amount of energy may push

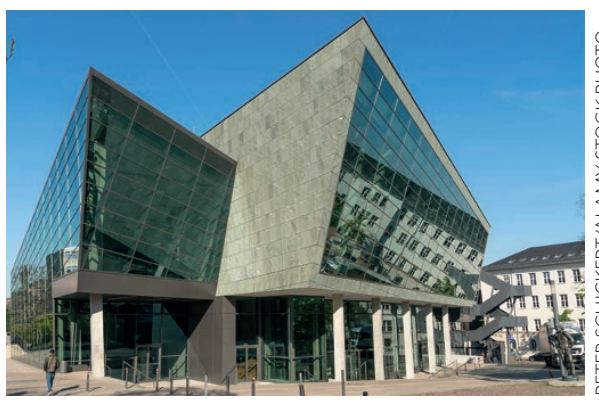

The Darmstadtium conference centre in Darmstadt holds a copy of the publication reporting the discovery of its eponymous element in its cornerstone.

the fusing system across a complex potential energy landscape. This concept - which had also constituted part of a controversial debate within the GSI group during measurement of the ${ }^{266} \mathrm{Hs}$ excitation function ${ }^{3}$ - had failed here, however.

Experimental superheavy-element chemistry is an extremely challenging undertaking and other, even heavier elements seem to promise more exciting features than darmstadtium. Therefore, this chemistry has not been attempted yet and only theoretical predictions of the chemical properties of darmstadtium exist. They point to a different ground-state configuration than that of its lighter homologues in group 10 , but nevertheless a rather similar chemical behaviour. As for all the superheavy elements, relativistic effects are of major importance here. These effects arise from the acceleration of the inner electrons to the highest velocities in the strong Coulomb field created by the many protons (here 110) in the heavy nucleus ${ }^{4}$.

In contrast to its chemistry, the physical properties of darmstadtium - or rather of its isotopes - have been experimentally studied, revealing some exciting nuclear structure features. The known darmstadtium isotopes sit on the edge of a region of deformed nuclei in the Segrè chart - the representation of nuclides as a function of their proton and neutron numbers. This region is characterized by enhanced nuclear stability at atomic number 108 (hassium), and at neutron numbers 152 and 162 , which are often called deformed sub-shell closures $^{5}$. Towards higher atomic numbers and higher mass, theory predicts that nuclear deformation vanishes and the area of spherical nuclei, which should be stabilized by quantum mechanical effects - referred to as the 'island of stability' - will be reached ${ }^{6}$.

Its nuclear deformation is also the cause of a striking feature of one of its isotopes, ${ }^{270} \mathrm{Ds}$, which represents the heaviest case of a metastable state - referred to as a $K$ isomer. This state resembles a wobble stone with an axis of total spin, which is inclined with respect to its symmetry axis, and whose decay is 'forbidden' by quantum mechanics. It is more stable than the ground state - an uncommon feature for nuclei. This is possibly a first hint of some interesting physics, yet to be discovered, at the onset of the development towards the nuclear sphericity predicted to occur in this area. In addition, these metastable states, depending on deformation, have the potential to be tracers guiding us towards the "island of stability".

These interesting features make ${ }^{270} \mathrm{Ds}$ one of the cornerstones in the arc leading to the superheavy elements.

DIETER ACKERMANN is at the Alternative Energies and Atomic Energy Commission, at the Large Heavy Ion National Accelerator Centre (GANIL), CEA-CNRS, Bd. Becquerel, 55027, F-14076 Caen, France. He is at present also supported by the European Commission in the framework of the CEA-EUROTALENT programme. e-mail: Dieter.Ackermann@ganil.fr

\section{References}

1. Hofmann, S. et al. Z. Phys. A 350, 277-280 (1995)

2. Münzenberg, G. et al. in GSI Scientific Report 1985, GSI Report 1986-1 (ed. Grundinger, U.) 29 (GSI, 1986).

3. Hofmann, S. On Beyond Uranium: Journey to the End of the Periodic Table (Taylor \& Francis, 2002).

4. Türler, A. \& Pershina, V. Chem. Rev. 113, 1237-1312 (2013).

5. Ackermann, D. \& Theisen, Ch. Phys. Scr. 92, 083002 (2017).

6. Cwiok, S., Heenen, P.-H. \& Nazarewicz, W. Nature 433, 705-709 (2005)

7. Ackermann, D. Nucl. Phys. A 944, 376-387 (2015).

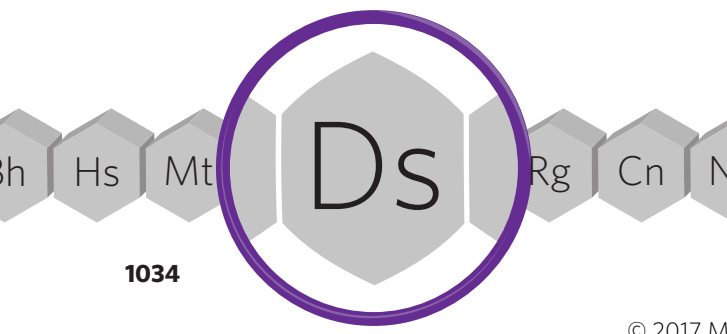

(5) 